\title{
Scattering of Conduction Electrons by a Ferromagnetic Domain Wall *
}

\author{
Masanori Yamanaka and Tohru Koma ${ }^{1}$ \\ Department of Applied Physics, Science University of Tokyo, Kagurazaka, Shinjuku-ku, Tokyo \\ 162, Japan \\ ${ }^{1}$ Department of Physics, Gakushuin University, Mejiro, Toshima-ku, Tokyo 171, Japan
}

\begin{abstract}
We study the scattering of an electron by a ferromagnetic domain wall of the quantum Heisenberg-Ising model (XXZ model) with certain boundary conditions. The spin of the electron interacts with the spins of the XXZ model by the Hund coupling. Using the exact domain wall ground states of the XXZ model, we analytically obtain the exact effective Schrödinger equation for conduction electrons. This equation coincides with a conventional phenomenological Schrödinger equation which was derived in a classical treatment of spins of a domain wall. By solving the Schrödinger equation numerically, we have calculated the transmission coefficient which is a function of the Hund coupling and of the anisotropy of the XXZ model. It turns out that the transmission coefficient is vanishing in the low energy limit for the electron.
\end{abstract}

Key words: Ferromagnetic metals, ferromagnetic domain walls, magnetoresistance, quantum Heisenberg-Ising model, XXZ model, domain wall ground states

\section{INTRODUCTION}

The early experiments of ferromagnetic materials [1] show that the resistivity increases with the increase of the number of the domain walls. This result seems to admit of no doubt because the conduction electrons are scattered by the domain walls [2]. An opposite result, however, was reported recently, i.e., the existence of a negative domain wall contribution to the resistivity was proposed from experimental [3-5] and theoretical [6] studies. In particular, Tatara and Fukuyama [6] stated that the existence of impurities plays an important roll for the negative domain wall contribution. We should note that some other recent studies [7-10] show that a domain wall contribution to resistivity is positive. As a starting point, it is necessary to study the problem of the scattering of conduction electrons by a single

\footnotetext{
*Proceedings of the 4th International Symposium on Physics of Magnetic Materials (ISPMM'98), August 1998, Sendai Japan.
} 
domain wall. This problem was considered in the early works [2] by Cabrera and Falicov. But they treated the spins of the domain wall classically.

In this paper, we study the scattering of an electron by a quantum domain wall which is realized as a ground state of the quantum Heisenberg-Ising model (XXZ model) with certain boundary conditions [11 14]. The spin of the conduction electron interacts with the spins of the XXZ model by the Hund coupling. Our main results are the following two: (i) We analytically obtain the exact effective Schrödinger equation for the conduction electron. More precisely the spin of the conduction electron feels the effective magnetic field of $\tanh (x / \lambda)$, and the amplitude of the spin flip is proportional to $1 / \cosh (x / \lambda)$. Here $x$ is the one-dimensional coordinate perpendicular to the domain wall centered at $x=0$, and $\lambda$ is the width of the domain wall. Our effective Schrödinger equation coincides with a conventional phenomenological Schrödinger equation by Cabrera and Falicov [2]. From this equation, one can immediately notice that the domain wall never transmits an electron with low energy without a spin flip of the electron. (ii) By solving the effective Schrödinger equation numerically, we have calculated the transmission coefficient which is a function of the Hund coupling and of the width of the domain wall. (The width is determined by the anisotropic exchange of the XXZ model.) It turns out that the transmission coefficient is vanishing in the low energy limit for the electron. As an example of a real system, consider a quantum wire with a single domain and with low density impurities. Then the transmission coefficient we obatined leads to the conductance by relying on the Landauer formula. Unfortunately it is not so easy to realize this situation in an experiment.

Throughout the present paper, we assume that the systems we consider are spatially homogeneous except for the $x$ direction perpendicular to domain walls. Having this assumption in mind, we treat the systems in any dimension as a one-dimensional one in this paper.

\section{A CLASSICAL DOMAIN WALL}

Before discussing a quantum domain wall, we briefly review a classical treatment of a domain wall. The spin configuration of a classical domain wall (Bloch wall) is represented by a vector-valued function $\vec{M}(x)$ of the position $x$. In [2], the authors assumed $\vec{M}(x)=B(-\operatorname{sech}(x / \lambda), 0,-\tanh (x / \lambda))$, where $\lambda$ is the width of the domain wall, and $B$ is a real constant. The interaction between $\vec{M}(x)$ and the spin $\vec{s}=\left(s^{(1)}, s^{(2)}, s^{(3)}\right)$ of a conduction electron is given by the Hund coupling $\left(M^{(1)}(x) s^{(1)}+M^{(2)} s^{(2)}+\Delta M^{(3)} s^{(3)}\right)$ with the anisotropy $\Delta$. Then the Schrödinger equation for the conduction electron is given by

$$
\left\{\begin{array}{l}
{\left[-\frac{\hbar^{2}}{2 m} \frac{d^{2}}{d x^{2}}-\frac{B \Delta}{2} \tanh \left(\frac{x}{\lambda}\right)-E\right] \psi_{\uparrow}=\frac{B}{2} \operatorname{sech}\left(\frac{x}{\lambda}\right) \psi_{\downarrow},} \\
{\left[-\frac{\hbar^{2}}{2 m} \frac{d^{2}}{d x^{2}}+\frac{B \Delta}{2} \tanh \left(\frac{x}{\lambda}\right)-E\right] \psi_{\downarrow}=\frac{B}{2} \operatorname{sech}\left(\frac{x}{\lambda}\right) \psi_{\uparrow},}
\end{array}\right.
$$

where $\psi_{\uparrow}$ and $\psi_{\downarrow}$ are, respectively, the up $(\uparrow)$ and the down $(\downarrow)$ components of the wavefunction of the electron, and $m$ is the mass of the electron. In the region far right from the domain wall $(x \sim \infty)$, a up-spin electron has lower energy of the Hund coupling than an 
down-spin electron. In the opposite region $(x \sim-\infty)$, up and down spins interchange their situation. If an electron goes thorough the domain wall without a spin flip of the electron, then the electron feels the potential barrier. A spin flip of the electron occurs only near the domain wall.

In this treatment, the effective magnetic potential for the conduction electrons is assumed to be identical to the profile of the domain wall. Clearly the potential should be derived from a microscopic Hamiltonian because the spins of the conduction electrons interact with quantum spins of a domain wall. The domain wall also should be realized in the microscopic Hamiltonian. Thus the phenomenological Schrödinger equation by Cabrera and Falicov needs to be justified from a microscopic level.

\section{A QUANTUM DOMAIN WALL}

In order to introduce a quantum domain wall, we consider the spin- $1 / 2$ Heisenberg-Ising model (XXZ model) on the one-dimensional lattice $[-L, L]$. The Hamiltonian is given by

$$
H_{\mathrm{dw}}=-J_{H} \sum_{y=-L}^{L-1}\left(S_{y}^{(1)} S_{y+1}^{(1)}+S_{y}^{(2)} S_{y+1}^{(2)}+\Delta_{H} S_{y}^{(3)} S_{y+1}^{(3)}\right)+\frac{J_{H}}{2} \sqrt{\left(\Delta_{H}\right)^{2}-1}\left[S_{-L}^{(3)}-S_{L}^{(3)}\right]
$$

where $\vec{S}_{y}=\left(S_{y}^{(1)}, S_{y}^{(2)}, S_{y}^{(3)}\right)$ is the spin-1/2 operator at the site $y \in[-L, L]$, and $J_{H}, \Delta_{H}$ are the exchange integral and the anisotropy, respectively. We assume $J_{H}>0$ and $\Delta_{H}>1$. We apply the boundary fields to make a single domain wall as a ground state. The sector of the ground states [11]14] of $H_{\mathrm{dw}}$ for a finite size $L$ is spanned by the set of the product states 12

$$
\Psi(z)=\left|\eta_{-L}(z), \eta_{-L+1}(z), \ldots, \eta_{L-1}(z), \eta_{L}(z)\right\rangle
$$

where $z$ is a complex number, and

$$
\eta_{y}(z)=\frac{1}{\sqrt{1+|z|^{2} q^{2 y}}}\left(|\uparrow\rangle_{y}+z q^{y}|\downarrow\rangle_{y}\right)
$$

Here $q$ is defined as $\Delta_{H}=\left(q+q^{-1}\right) / 2$ with $0<q<1$, and $|\uparrow\rangle$ and $|\downarrow\rangle$ are the spin up and down states, respectively. Write $z=e^{\mu+i \phi}$ with real numbers $\mu, \phi$. Then $\mu$ specifies the position of the domain wall and the angle $\phi$ is a quantum mechanical phase corresponding to the degrees of freedom of the rotation about the third axis of the spin. For simplicity we set $z=1$. Then we have, from (四),

$$
\eta_{y}(z) \sim \begin{cases}|\uparrow\rangle_{y} & \text { for } y \sim \infty \\ |\downarrow\rangle_{y} & \text { for } y \sim-\infty\end{cases}
$$

in the thermodynamic limit $L \rightarrow+\infty$, and from (3),

$$
\left\langle\Psi(z), S_{y}^{(3)} \Psi(z)\right\rangle=\frac{1}{2} \tanh \left(\frac{x}{\lambda}\right) \quad \text { with } \lambda=-\frac{a}{\log q},
$$

where $a$ is the lattice constant, and we have used $x=a y$. Thus the expectation about the quantum domain wall ground state $\Psi(z)$ gives the profile of the classical domain wall. 


\section{A HEISENBERG-KONDO MODEL}

Now we consider the problem of the scattering of a conduction electron by a single quantum domain wall. To treat this problem, we introduce a Heisenberg-Kondo model. See Fig. 1 for the schematic structure. The Hamiltonian is given by

$$
H=H_{\mathrm{el}}+H_{\mathrm{dw}}+H_{\mathrm{el}-\mathrm{dw}}
$$

with the kinetic energy of the conduction electron

$$
H_{\mathrm{el}}=-\frac{\hbar^{2}}{2 m} \frac{d^{2}}{d x^{2}}
$$

and the Hund coupling

$$
H_{\mathrm{el}-\mathrm{dw}}=-J_{K} \sum_{y=-L}^{L} \mathcal{P}\left[\vec{s} \cdot \vec{S}_{y}+\left(\Delta_{K}-1\right) s^{(3)} S_{y}^{(3)}\right] \mathcal{P} \times \chi_{y}(x),
$$

between the spin $\vec{s}$ of the electron and the spins $\vec{S}_{y}$ of the domain wall of $H_{\mathrm{dw}}$ of (2)). Here $J_{K}, \Delta_{K}$ are real parameters, $\mathcal{P}$ is the projection operator onto the sector spanned by the ground states of $H_{\mathrm{dw}}$, and $\chi_{y}(x)$ is a characteristic function defined by

$$
\chi_{y}(x)= \begin{cases}1, & \text { if } x \in a[y, y+1] \\ 0, & \text { otherwise }\end{cases}
$$

By introducing the projection $\mathcal{P}$, we have neglected the excitations above the domain wall ground states of the XXZ model. In pure one dimension, this approximation is justified because of the energy gap above those ground states [14. But, in higher dimensions there exist gapless excitations above the domain wall ground states [13,14]. The treatment of the excitations is left for future studies [15].

Using the domain wall ground state $\Psi(z)$ of (3), a single-electron "eigenstate" can be written as

$$
\Phi(z)=\left(\psi_{\uparrow}|\uparrow\rangle+\psi_{\downarrow}|\downarrow\rangle\right) \otimes \Psi(z)
$$

where $\psi_{\sigma}(x)$ is the $\sigma$ component of the wave function of the conduction electron. Then the effective Schrödinger equation for the conduction electron is given by

$$
\langle\Psi(z), H \Phi(z)\rangle=E\langle\Psi(z), \Phi(z)\rangle,
$$

where we have used the single-electron Schrödinger equation $H \Phi=E \Phi$ for the Hamiltonian $H$ of (7) and with the energy eigenvalue $E$. Using (6) and $2\left\langle\Psi(z), S_{y}^{( \pm)} \Psi(z)\right\rangle=\operatorname{sech}(x / \lambda)$, the equation (12) is written as

$$
\left\{\begin{array}{l}
{\left[-\frac{\hbar^{2}}{2 m} \frac{d^{2}}{d x^{2}}-\frac{J_{K} \Delta_{K}}{4} \tanh \left(\frac{x}{\lambda}\right)-E\right] \psi_{\uparrow}=\frac{J_{K}}{4} \operatorname{sech}\left(\frac{x}{\lambda}\right) \psi_{\downarrow},} \\
{\left[-\frac{\hbar^{2}}{2 m} \frac{d^{2}}{d x^{2}}+\frac{J_{K} \Delta_{K}}{4} \tanh \left(\frac{x}{\lambda}\right)-E\right] \psi_{\downarrow}=\frac{J_{K}}{4} \operatorname{sech}\left(\frac{x}{\lambda}\right) \psi_{\uparrow}}
\end{array}\right.
$$


This coincides with the phenomenological equation (1]) by Cabrera and Falicov. Although the vector (11) is not an eigenvector of the Hamiltonian $H$ of (7) in a usual sense, we can justify the above treatment for the present system in any dimension [15]. Suppose that an $\uparrow$ electron comes from the right side of the domain wall. Then the spin of the electron feels the potential barrier $-\left(J_{K} \Delta_{K} / 4\right) \tanh (x / \lambda)$ and flips with the transition amplitude $-\left(J_{K} / 4\right) \operatorname{sech}(x / \lambda)$ near the domain wall. Since the height of the potential wall is order of $J_{K}$, a conduction electron with low energy $\left(E<J_{K} \Delta_{K} / 4\right)$ never goes to the other side without a spin flip.

\section{NUMERICAL RESULTS AND DISCUSSION}

We solved the Schrödinger equation (13) numerically and obtained the transmission coefficient as a function of the energy $E$ of the conduction electron and of the width $\lambda$ of the domain wall. The results for the transmission coefficients with a spin flip are shown in Fig. 2. The remarkable feature is that the transmission coefficients are vanishing in the low energy limit for the conduction electron. As we expected, our results indicate that there appears no negative domain wall contribution to resistivity without introducing a new mechanism such as an effective interaction between domain walls and impurities. Actually such mechanisms were proposed by some authors [6, 10] for explaining the recent experiments [3 5,7,8]. However, it is very difficult to take account of the effect of impurities in a mathematically rigorous manner as in the present paper.

Acknowledgements The authors are grateful to Koh Mibu, Taro Nagahama, Naoto Nagaosa, Yoshiko Nakamura, Yoshichika Otani, Teruya Shinjo, and Gen Tatara for useful discussions. A part of the numerical calculations was performed by using facilities of the supercomputing center in ISSP, University of Tokyo. 


\section{REFERENCES}

[1] G.R. Taylor, S. Isin, and R.V. Coleman, Phys. Rev. 165, 621 (1968).

[2] G.G. Cabrera and L.M. Falicov, Status Solidi (b) 61, 539 (1974); ibid 62217 (1974).

[3] K. Hong and N. Giordano Phys. Rev. B51, 9855 (1995); J. Magn. Magn. Mat. 151, 396 (1995); J. Phys. Condens. Matt. 8, L301 (1996); ibid 10, L401 (1998).

[4] Y. Otani, K. Fukamichi, O. Kitakami, Y. Shimada, B. Pannetier, J.-P. Nozieres, T. Matsuda, and A. Tonomura, Proceedings of MRS Spring meeting, (San Francisco) 475, 215 (1998).

[5] U. Ruediger, J.Yu. Zhang, A.D. Kent, and S.S.P. Parkin, Phys. Rev. Lett., 80, 5639 (1998).

[6] G. Tatara and H. Fukuyama, Phys. Rev. Lett. 78, 3773 (1997).

[7] J.F. Gregg, W. Allen, K. Ounadjela, M. Viret, M. Hehn, S.M. Thompson, and J.M.D.Coey, Phys. Rev. Lett. 77, 1580 (1996).

[8] K. Mibu, T. Nagahama, and T. Shinjo, J. Magn. Magn. Mater. 16375 (1996); K. Mibu, T. Nagahama, and T. Ono, T. Shinjo, unpublished.

[9] M. Yamanaka and N. Nagaosa, J. Phys. Soc. Jpn. 65, 3088 (1996).

[10] P.M. Levy and S. Zhang, Phys. Rev. Lett. 79, 5110 (1997)

[11] F.C. Alcaraz, S.R. Salinas, and W.F. Wreszinski, Phys. Rev. Lett. 75, 930 (1993).

[12] C.-T. Gottstein and R.F. Werner, cond-mat/9501123.

[13] T. Matsui, Lett. Math. Phys. 37, 397 (1996); ibid 42, 229 (1997).

[14] T. Koma and B. Nachtergaele, Lett. Math. Phys. 40, 1 (1997); RIMS Kokyuroku (Kyoto University, Kyoto, Japan) No. 1035, 133 (1998); Adv. Theor. Math. Phys. 2, (1998), to appear.

[15] M. Yamanaka and T. Koma, in preparation. 


\section{FIGURES}

FIG. 1. A schematic description of the Heisenberg-Kondo model. The open circles denote the spins of the XXZ model. The conduction electron moves along the dotted line. The bonds of the solid lines denote the ferromagnetic exchange interactions of the XXZ model. The broken lines denote the Hund couplings between the spins of the XXZ model and the spin of the conduction electron.

FIG. 2. The transmission coefficients as a function of the energy $E$ of the conduction electron in the unit of $J_{K} \Delta_{K}$. 